Article

\title{
Input Disturbance Suppression for Unidirectional Matrix Converter with a Stability-Enhancing Modulation Scheme
}

\author{
Jiaxing Lei ${ }^{1,2, * \mathbb{D}}$, Chaofan Wei ${ }^{1,2}$ and Shuang Feng ${ }^{1,2}$ \\ 1 School of Electrical Engineering, Southeast University, Nanjing 210096, China \\ 2 Jiangsu Provincial Key Laboratory of Smart Grid Technology and Equipment, Southeast University, \\ Nanjing 210096, China \\ * Correspondence: jxlei@seu.edu.cn; Tel.: +86-025-83792260
}

Received: 13 June 2019; Accepted: 6 July 2019; Published: 8 July 2019

\begin{abstract}
This paper proposes an input voltage disturbance suppression control strategy for the unidirectional matrix converter (UMC) with a new modulation scheme enhancing the stability. In the new scheme, the modulation index is directly, rather than reversely, proportional to the instantaneous amplitude of input filter capacitor voltages. Contrary to traditional schemes, the stability of the UMC with this new scheme is even better with the increase of the transferred active power, which is particularly suitable for applications with sinusoidal and balanced input conditions. As to the disturbed input conditions, the new scheme could introduce low-frequency harmonics into output currents. To address this issue, a feedback control strategy of output current amplitude is further proposed to eliminate the additional harmonics. Stability analysis of a UMC with the proposed modulation scheme and feedback control strategy is presented. Experimental results have verified the validity of the proposed control solution.
\end{abstract}

Keywords: matrix converter; stability; feedback control; input voltage disturbance

\section{Introduction}

The matrix converter $(\mathrm{MCs})$ realizes direct $\mathrm{AC}-\mathrm{AC}$ power conversion with minimal passive components [1] and has attracted attention from many researchers [2-5]. The indirect matrix converter (IMC) is a subtype of the MC [6], which consists of a rectifier stage and an inverter stage, as shown in Figure 1a. The IMC features a simple commutation strategy which could achieve zero current switching for the rectifier stage. For applications where only unidirectional power supply is required, such as the aerospace power supply system, the rectifier stage of the IMC could be simplified by removing the forward transistors. Termed as the unidirectional MC (UMC) in this paper, the simplified topology is shown in Figure 1b. Moreover, it is possible to further reduce the usage of transistors by using other configurations of switches at the rectifier stage [6-9].

Due to the lack of intermediate energy storage elements, the $M C$ is sensitive to input voltage disturbances. Besides, LC filter resonance could be easily affected if the damping is insufficient. Both issues are related with the modulation scheme of the MC. Most existing modulation schemes address the first issue effectively, sampling instantaneous input voltages to correct the modulation signals [10-13]. Specifically, the modulation index of the MC is obtained from the reference output voltage amplitude divided by the instantaneous input voltage amplitude, which is designated as the feedforward control. 


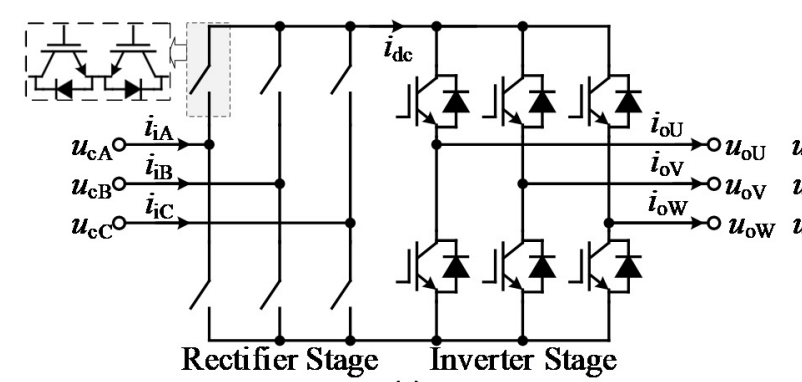

(a)

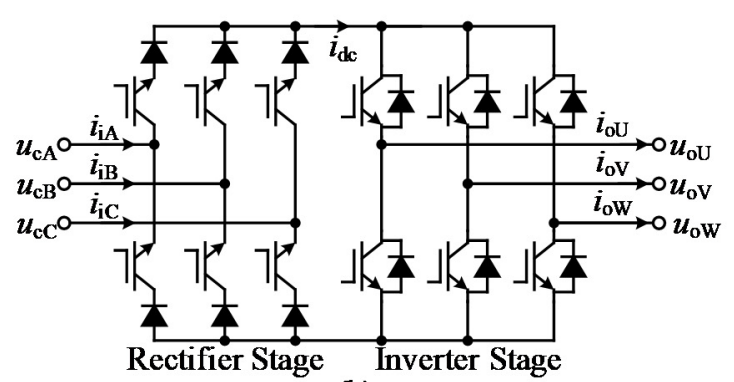

(b)

Figure 1. Topologies of the indirect matrix converter (a) and the unidirectional matrix converter (UMC) (b).

Nevertheless, the stability issue has subordinate priority in the control goal of existing schemes. If the input source is ideal, source voltages can be sampled for modulation so that the MC stability would not be affected. Yet, the damping of the input LC filter is insufficient, and thus additional passive or active damping control is still necessary [14]. When the source is weak with high impedance (such as a generator input), the filter capacitor voltages are sampled for modulation. In this case, the stability of MC could be deteriorated, as is well acknowledged in the literature. In [15], the small-signal analysis shows that these schemes lead to unstable poles. In [16], the phase margin is found to be negative. Reference [17] reveals that these schemes generate negative input admittances, which is the reason for the worsened stability. In addition, the analyses in the literature also prove that the higher the transferred active power is, the worse the stability will be.

To improve the stability of the MC with existing modulation schemes, some stabilization methods have been proposed in the literature, most of which adopt digitally filtered input voltages to calculate the modulation signals [16-19]. Though they do have the capability to stabilize the MC, their performance relies on the parameters of the digital filters [17]. Besides, the digital filter directly transfers the input voltage disturbances to output voltages, degrading the output control performance [20]. Therefore, structure and parameters of the digital filters need to be carefully designed, considering both the stability improvement and disturbance suppression.

In this paper, a novel control solution is proposed, especially applicable to the UMC. Different from existing solutions, the proposed solution grants higher priority to the stability issue and adopts additional methods to suppress the effects of input voltage disturbances, if any. A new modulation scheme is firstly applied to the UMC, in which the modulation index is directly proportional to the instantaneous amplitude of input filter capacitor voltages. The new scheme generates positive input admittances, which become larger with the increase of the active power. Therefore, it always enhances the stability of the UMC without additional stabilization methods, which is particularly suitable for situations with balanced and sinusoidal input voltages. When the input voltages are unbalanced and/or distorted, low-frequency harmonics are present in output currents. A feedback control strategy of output current amplitude is then proposed to eliminate the unwanted harmonics. Stability analysis is performed for the UMC with the proposed control solution. Experimental results are provided to verify the effectiveness of the proposed control solution.

\section{Modulation Schemes for the UMC}

\subsection{Traditional Modulation Scheme}

Control of the typical UMC system is shown in Figure 2. The load of the UMC can be a simple passive load, as adopted in this paper, or an active load such as a motor, which does not affect the effectiveness of the study in this paper. As discussed in [17], the load effect has quite limited effect on the input stability of the MC, and thus can be ignored for simplifying the stability study of the MC $[16,17]$. The output control, such as the typical field-oriented control for the motor, generates the reference amplitude, $u_{\mathrm{om}}{ }^{*}$, and phase angle, $\theta_{\mathrm{ou}}$, of the output voltages of the UMC. 


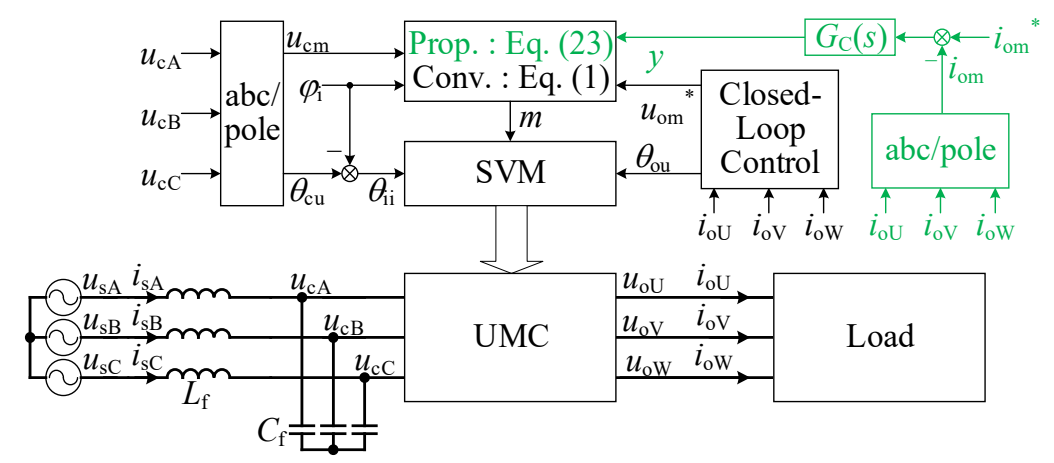

Figure 2. Control of the typical UMC system. Space vector modulation (SVM) is applied to generate the switching signals of UMC.

For traditional modulation schemes with feedforward control, the modulation index, $m$, of the $\mathrm{UMC}$ is calculated as

$$
m=\frac{2 u_{\mathrm{om}}^{*}}{\sqrt{3} u_{\mathrm{cm}} \cos \varphi_{\mathrm{i}}}
$$

where $u_{\mathrm{om}}{ }^{*}$ is the reference output voltage amplitude, $u_{\mathrm{cm}}$ is the instantaneous amplitude of the input filter capacitor voltages, and $\varphi_{\mathrm{i}}$ is the input power factor angle. The phase angle, $\theta_{\mathrm{ii}}$, for the rectifier stage modulation is the subtraction of $\theta_{\mathrm{cu}}$ and $\varphi_{\mathrm{i}}$, where $\theta_{\mathrm{cu}}$ is the phase angle of the capacitor voltages.

Both the duty cycle calculation and the switching pattern for the UMC are the same as those for the typical indirect matrix converter [2], which are not changed by the proposed modulation scheme and the feedback control strategy. As presented below, the proposed modulation scheme only changes the expression of the modulation index.

With the traditional modulation scheme, the $\alpha \beta$-axis components of input currents can be expressed as [14]

$$
\begin{aligned}
& i_{\mathrm{i} \alpha}=m \cos \theta_{\mathrm{ii}} i_{\mathrm{dc}}=m \cos \left(\theta_{\mathrm{cu}}-\varphi_{\mathrm{i}}\right) i_{\mathrm{dc}}, \\
& i_{\mathrm{i} \beta}=m \sin \theta_{\mathrm{ii}} i_{\mathrm{dc}}=m \sin \left(\theta_{\mathrm{cu}}-\varphi_{\mathrm{i}}\right) i_{\mathrm{dc}} .
\end{aligned}
$$

The $\alpha \beta$-axis components of capacitor voltages satisfy

$$
\begin{aligned}
& u_{\mathrm{c} \alpha}=u_{\mathrm{cm}} \cos \theta_{\mathrm{cu}} \\
& u_{\mathrm{c} \beta}=u_{\mathrm{cm}} \sin \theta_{\mathrm{cu}} .
\end{aligned}
$$

Variable $i_{\mathrm{dc}}$ in Equation (2) is defined as [14]

$$
i_{\mathrm{dc}}=\frac{\sqrt{3}\left(u_{\mathrm{o} \alpha}^{*} i_{\mathrm{o} \alpha}+u_{\mathrm{o} \beta}^{*} i_{\mathrm{o} \beta}\right)}{2 u_{\mathrm{om}}^{*}} .
$$

With the substitution of Equations (1), (4), and (6), Equation (2) can be rewritten as

$$
\begin{aligned}
& i_{\mathrm{i} \alpha}=\frac{p_{\mathrm{o}}\left(u_{\mathrm{c} \alpha}+u_{\mathrm{c} \beta} \tan \varphi_{\mathrm{i}}\right)}{1.5 u_{\mathrm{cm}}^{2}}, \\
& i_{\mathrm{i} \beta}=\frac{p_{\mathrm{o}}\left(u_{\mathrm{c} \beta}-u_{\mathrm{c} \alpha} \tan \varphi_{\mathrm{i}}\right)}{1.5 u_{\mathrm{cm}}^{2}},
\end{aligned}
$$

where $p_{\mathrm{o}}$ is the instantaneous active power to be transferred to the load, expressed as

$$
p_{\mathrm{o}}=1.5\left(u_{\mathrm{o} \alpha}^{*} i_{\mathrm{o} \alpha}+u_{\mathrm{o} \beta}^{*} i_{\mathrm{o} \beta}\right),
$$


where $u_{\mathrm{o} \alpha}{ }^{*}$ and $u_{\mathrm{o} \beta}{ }^{*}$ are the reference $\alpha \beta$-axis components of the output voltages. Theoretically, $p_{\mathrm{o}}$ should be a constant DC signal for the balanced and sinusoidal load.

Usually, the stability analysis for the MC system should be performed in the synchronous reference frame, where all the variables are DC signals. In the synchronous reference frame oriented with the source voltage vector, the dq-axis input currents are obtained from Equations (7) and (8) as

$$
\begin{aligned}
& i_{\mathrm{id}}=\frac{p_{\mathrm{o}}\left(u_{\mathrm{cd}}+u_{\mathrm{cq}} \tan \varphi_{\mathrm{i}}\right)}{1.5 u_{\mathrm{cm}}^{2}}, \\
& i_{\mathrm{iq}}=\frac{p_{\mathrm{o}}\left(u_{\mathrm{cq}}-u_{\mathrm{cd}} \tan \varphi_{\mathrm{i}}\right)}{1.5 u_{\mathrm{cm}}^{2}},
\end{aligned}
$$

where the square of the instantaneous amplitude of capacitor voltages satisfies

$$
u_{\mathrm{cm}}^{2}=u_{\mathrm{cd}}^{2}+u_{\mathrm{cq}}^{2}
$$

where $u_{\mathrm{cd}}$ and $u_{\mathrm{cq}}$ are the dq-axis components of capacitor voltages.

The stability analysis method based on the input impedance is quite intuitive and thus is adopted in this paper. According to the definition of input admittances of the MC [17], the dq-axis input admittances with the traditional modulation scheme are

$$
\begin{gathered}
Y_{\mathrm{id}}=\frac{\partial i_{\mathrm{id}}}{\partial u_{\mathrm{cd}}} \approx-\frac{P_{o}}{1.5 U_{c m}^{2}} \\
Y_{\mathrm{iq}}=\frac{\partial i_{\mathrm{iq}}}{\partial u_{\mathrm{cq}}} \approx \frac{P_{\mathrm{o}}}{1.5 U_{\mathrm{cm}}^{2}}
\end{gathered}
$$

where the capitalized variables represent the steady-state values. The detailed derivation of Equations (13) and (14) is presented in the Appendix A section. Equation (13) shows that the $\mathrm{d}$-axis admittance $Y_{\text {id }}$ is always negative, which is the reason for the deteriorated stability of the UMC with the traditional modulation scheme. Besides, the larger the active power $P_{\mathrm{o}}$, the larger the absolute value of $Y_{\mathrm{id}}$ and the worse the stability of UMC.

\subsection{Stability-Enhancing Modulation Scheme}

From the derivation process of the input admittances, the instantaneous amplitude, $u_{\mathrm{cm}}$, located in the denominator of the modulation index expression in Equation (1) is the reason for the negative sign of $Y_{\text {id }}$. Therefore, a new modulation scheme is inspired, which calculates the modulation index with

$$
m=\frac{2 u_{\mathrm{om}}^{*} u_{\mathrm{cm}}}{\sqrt{3} u_{\mathrm{cm}}^{2} \cos \varphi_{\mathrm{i}}}
$$

It shows that $m$ is directly, rather than inversely, as in the traditional modulation schemes, proportional to $u_{\mathrm{cm}}$. Theoretically, the modulation index obtained with Equation (15) is the same with that obtained with Equation (1) at the steady state, if the system remains stable. This is because $U_{\mathrm{cm}}$ in Equation (15) is exactly the same as the steady-state value of $u_{\mathrm{cm}}$. However, the stability performance with each of the two equations differs significantly, as discussed below.

With the substitution of Equations (6) and (15), the $\alpha \beta$-axis components of input currents are obtained from Equation (2):

$$
i_{\mathrm{i} \alpha}=\frac{p_{\mathrm{o}}\left(u_{\mathrm{c} \alpha}+u_{\mathrm{c} \beta} \tan \varphi_{\mathrm{i}}\right)}{1.5 U_{\mathrm{cm}}^{2}},
$$




$$
i_{\mathrm{i} \beta}=\frac{p_{\mathrm{o}}\left(u_{\mathrm{c} \beta}-u_{\mathrm{c} \alpha} \tan \varphi_{\mathrm{i}}\right)}{1.5 U_{\mathrm{cm}}^{2}} .
$$

Accordingly, the dq-axis components of input currents with this new scheme are

$$
\begin{aligned}
& i_{\mathrm{id}}=\frac{p_{\mathrm{o}}\left(u_{\mathrm{cd}}+u_{\mathrm{cq}} \tan \varphi_{\mathrm{i}}\right)}{1.5 U_{\mathrm{cm}}^{2}} \\
& i_{\mathrm{iq}}=\frac{p_{\mathrm{o}}\left(u_{\mathrm{cq}}-u_{\mathrm{cd}} \tan \varphi_{\mathrm{i}}\right)}{1.5 U_{\mathrm{cm}}^{2}}
\end{aligned}
$$

and the dq-axis input admittances can be derived as

$$
\begin{gathered}
Y_{\mathrm{id}}=\frac{\partial i_{\mathrm{id}}}{\partial u_{\mathrm{cd}}} \approx \frac{P_{o}}{1.5 U_{c m}^{2}}, \\
Y_{\mathrm{iq}}=\frac{\partial i_{\mathrm{iq}}}{\partial u_{\mathrm{cq}}} \approx \frac{P_{\mathrm{o}}}{1.5 U_{\mathrm{cm}}^{2}} .
\end{gathered}
$$

For the UMC, the transferred active power $P_{\mathrm{o}}$ is positive, and thus both $Y_{\mathrm{id}}$ and $Y_{\mathrm{iq}}$ are positive. As a result, the new scheme always enhances the stability of the UMC, saving the need for an additional stabilization method. Besides, $Y_{\mathrm{id}}$ and $Y_{\mathrm{iq}}$ become larger in the positive direction with the increase of $P_{\mathrm{o}}$. Therefore, the stability of the UMC with this new scheme is even better under high power applications, contrary to existing schemes. This is a prominent advantage of the proposed modulation scheme.

It can be derived that the dq-axis impedances are both zero if the source voltages $u_{\mathrm{sA}}, u_{\mathrm{sB}}$, and $u_{\mathrm{SC}}$ are sampled for modulation, which thereby have no effect on the stability. Consequently, for traditional schemes, sampling the source voltages are preferred if they are accessible. However, an additional passive or active stabilization method is still necessary to suppress the filter resonance. On the contrary, sampling the capacitor voltages is preferred for the proposed scheme even if the source voltages are accessible, as the stability and filter damping can always be enhanced with this scheme.

\section{Proposed Feedback Control Strategy and Stability Analysis}

\subsection{Feedback Control of Output Current Amplitude}

In some cases, such as a generator input, the input voltages are sinusoidal and balanced. The proposed modulation scheme can be applied to achieve enhanced stability and satisfactory power quality without additional control, reducing the control complexity. Yet, when the input source is the utility grid, which could be unbalanced and/or distorted, the output power quality is reduced by the proposed scheme. The traditional modulation schemes with an additional stabilization method also suffer from this issue [20]. This issue can be addressed by incorporating an additional controller in the closed-loop control on the output side. This paper proposes a novel feedback control strategy for the proposed modulation scheme.

If Equation (15) is directly applied to the UMC, the actual output voltage amplitude $u_{\text {om }}$ is

$$
u_{\mathrm{om}}=\frac{\sqrt{3}}{2} m u_{\mathrm{cm}} \cos \varphi_{\mathrm{i}}=\frac{u_{\mathrm{om}}^{*} u_{\mathrm{cm}}^{2}}{U_{\mathrm{cm}}^{2}}
$$

It can be seen that $u_{\mathrm{om}}$ is not equal to its reference $u_{\mathrm{om}}{ }^{*}$, but is directly proportional to the square of $u_{\mathrm{cm}}$. Under disturbed input conditions, $u_{\mathrm{cm}}{ }^{2}$ is not a constant DC signal but contains rich low-frequency harmonics [20]. For example, for the unbalanced component, the $5^{\text {th }}$ and $7^{\text {th }}$ harmonics of the capacitor voltages introduce $2^{\text {nd }}, 4^{\text {th }}, 6^{\text {th }}$, and $8^{\text {th }}$ harmonics in $u_{\mathrm{cm}}{ }^{2}$. Consequently, $u_{\mathrm{om}}$ also 
contains low-frequency harmonics even if its reference $u_{\mathrm{om}}{ }^{*}$ is constant, directly degrading the output power quality.

To address this issue, the proposed feedback control strategy shown in Figure 2 can be applied, which adopts a controller, $G_{C}(s)$, to regulate the output current amplitude, $i_{\text {om }}$. The output signal of $G_{C}(s)$ is defined as $y$, which is used to further correct the modulation index:

$$
m=\frac{2 u_{\mathrm{om}}^{*} u_{\mathrm{cm}}}{\sqrt{3}(1-y) U_{\mathrm{cm}}^{2} \cos \varphi_{\mathrm{i}}} .
$$

The actual $u_{\text {om }}$ with the proposed feedback control strategy is

$$
u_{\mathrm{om}}=\frac{\sqrt{3} m u_{\mathrm{cm}} \cos \varphi_{\mathrm{i}}}{2}=u_{\mathrm{om}}^{*} \frac{u_{\mathrm{cm}}^{2}}{U_{\mathrm{cm}}^{2}(1-y)} .
$$

The signal flow graph of the proposed feedback control strategy is shown in Figure 3a. Its small-signal linearization is shown in Figure $3 b$. In Figure $3, G_{P}(s)$ is the transfer function from $u_{\mathrm{om}}$ to $i_{\mathrm{om}}$, which is approximately equal to $1 /\left(L_{\mathrm{o}} s+R_{\mathrm{o}}\right)$, where $L_{\mathrm{o}}$ and $R_{\mathrm{o}}$ are the output inductance and resistance, respectively. The closed-loop transfer function $H_{\mathrm{y}}(s)$ from $\left(1-u_{\mathrm{cm}}{ }^{2} / U_{\mathrm{cm}}{ }^{2}\right)$ to $y$ is obtained from Figure $3 \mathrm{~b}$ :

$$
H_{\mathrm{y}}(s)=\frac{u_{\mathrm{om}}^{*} G_{\mathrm{C}}(s) G_{\mathrm{P}}(s)}{1+u_{\mathrm{om}}^{*} G_{\mathrm{C}}(s) G_{\mathrm{P}}(s)}
$$

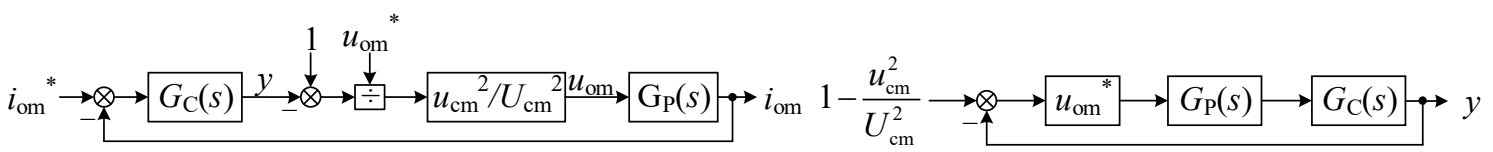

(a)

(b)

Figure 3. Signal flow graphs of the proposed feedback control strategy: (a) the large-signal control loop; (b) the small-signal control loop.

According to Equation (24), to eliminate the effects of input voltage disturbances, the low-frequency components in $(1-y)$ should be equal to $u_{\mathrm{cm}}{ }^{2} / U_{\mathrm{cm}}{ }^{2}$. Therefore, the complex gains of $H_{\mathrm{y}}(s)$ at the concerned frequencies associated with the input voltage disturbances should be at unity. To achieve this goal, several advanced resonant controllers (RCs) are adopted in parallel to construct the controller $G_{C}(s)$ :

$$
G_{\mathrm{C}}(s)=\sum \frac{K_{\mathrm{R} n}}{u_{\mathrm{om}}^{*}} \frac{s\left(L_{\mathrm{o}} s+R_{\mathrm{o}}\right)}{s^{2}+\left(n \omega_{\mathrm{i}}\right)^{2}}
$$

where $\omega_{\mathrm{i}}$ is the input angular frequency, $n$ is the order of the concerned frequency, and $K_{\mathrm{R} n}$ is the static gain of each RC. The integral controller can also be included in $G_{C}(s)$ by setting $n=0$, so that the effect of imprecise $U_{\mathrm{cm}^{2}}{ }^{2}$ in Equation (23) can be eliminated. Each RC compensates the additional loop gain $u_{\mathrm{om}}{ }^{*}$ and the pole of $G_{\mathrm{P}}(s)$ in order to simplify the expression of $H_{\mathrm{y}}(s)$ and improve the dynamic performance. According to the principle of $\mathrm{RC}$, it provides infinite gain at its central frequency but sorely limited gain at the other frequencies. It can be deduced that the complex gains of $H_{\mathrm{y}}(s)$ at the frequencies $n \omega_{\mathrm{i}}$ are at unity but approach zero at the other frequencies. Therefore, the proposed feedback control strategy can eliminate the effects of input voltage disturbances without introducing other harmonics.

\subsection{Stability Analysis}

Stability analysis is performed in this part to verify the validity of the proposed feedback control strategy. The impedance-based method presented in [17] is adopted to perform the analysis. To simplify 
the analysis, the dq-axis coupling terms and the load effect, which have quite limited effect on the stability, are ignored in this paper.

With the proposed feedback control strategy, the dq-axis components of input currents can be obtained from Equations (18) and (19) by replacing Equation (15) with Equation (23):

$$
\begin{aligned}
& i_{\mathrm{id}}=\frac{p_{\mathrm{o}}\left(u_{\mathrm{cd}}+u_{\mathrm{cq}} \tan \varphi_{\mathrm{i}}\right)}{1.5 U_{\mathrm{cm}}^{2}(1-y)}, \\
& i_{\mathrm{iq}}=\frac{p_{\mathrm{o}}\left(u_{\mathrm{cq}}-u_{\mathrm{cd}} \tan \varphi_{\mathrm{i}}\right)}{1.5 U_{\mathrm{cm}}^{2}(1-y)} .
\end{aligned}
$$

As signal $y$ is associated with $u_{\mathrm{cm}}{ }^{2}$, according to the principle of the proposed strategy, the input admittances of the UMC are only different with those with the new modulation scheme, which can be derived from Equations (27) and (28) with the substitution of $H_{\mathrm{y}}(s)$ :

$$
\begin{gathered}
Y_{\mathrm{id}}=\frac{\partial i_{\mathrm{id}}}{\partial u_{\mathrm{cd}}} \approx \frac{P_{\mathrm{o}}}{1.5 U_{\mathrm{cm}}^{2}}\left[1-2 H_{\mathrm{y}}(s)\right], \\
Y_{\mathrm{iq}}=\frac{\partial i_{\mathrm{iq}}}{\partial u_{\mathrm{cq}}} \approx \frac{P_{\mathrm{o}}}{1.5 U_{\mathrm{cm}}^{2}} .
\end{gathered}
$$

The detailed derivation is provided in the Appendix A section. Equations (29) and (30) show that the proposed strategy could theoretically influence the d-axis input admittance and further the UMC stability, subject to the transfer function $H_{\mathrm{y}}(s)$. Nevertheless, the practical influence is sorely limited, as analyzed below.

The small signal d-axis input circuit of the UMC is shown in Figure 4 , where $R_{\mathrm{f}}$ is the resistance of the filter inductor, $L_{\mathrm{f}}$. The transfer function $H_{\mathrm{is}}(s)$ from $\Delta i_{\mathrm{id}}$ to $\Delta i_{\mathrm{sd}}$ is obtained from Figure 4 :

$$
H_{\mathrm{is}}(s)=\frac{1}{1+\left(s C_{\mathrm{f}}+Y_{\mathrm{id}}\right)\left(s L_{\mathrm{f}}+R_{\mathrm{f}}\right)},
$$

The poles of $H_{\text {is }}(s)$ can be used to evaluate the UMC stability. If there is a pole located in the right-half complex plane, the UMC becomes unstable.

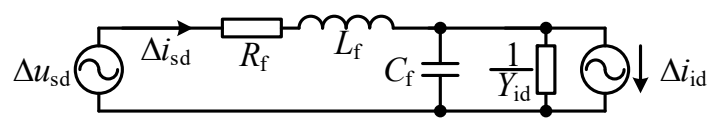

Figure 4. Small signal d-axis input circuit of the UMC.

As shown in Figure 5, the poles with the traditional modulation schemes are located at the unstable region with positive real parts, resulting from the negative input admittances. Therefore, an additional stabilization method is inevitably needed for traditional schemes so as to drive the unstable poles to the stable region, which requires great control effort.

The proposed modulation and control strategy are applied to the UMC instead. In this example, five RCs with $n=0,2,4,6$, and 8 are adopted in the controller $G_{C}(s)$, which are sufficient to eliminate the effects of the imprecise $U_{\mathrm{cm}}{ }^{2}$, the input voltage unbalance, and the common $5^{\text {th }}$ and $7^{\text {th }}$ input voltage harmonics. With the static gain $K_{\mathrm{R} n}$ increasing from 0 to 4000 , the pole locus of $H_{\mathrm{is}}(s)$ is illustrated in Figure 5. When the proposed feedback control strategy is disabled with $K_{\mathrm{R} n}=0$, the poles associated with the resonant mode of the input LC filter are located in the stable region and far away from the imaginary axis. This proves that the new modulation scheme enhances the stability dramatically. When $K_{\mathrm{R} n}$ increases, the damping of the resonant mode increases first and then decreases. When $K_{\mathrm{R} n}$ is very large, the poles may move to the right-half complex plane, showing that the UMC becomes unstable. The value of $K_{R n}$ at the critically stable point is about 2000, which far exceeds the value of 
200 adopted in practice. The poles with $K_{\mathrm{R} n}=200$ are highlighted in Figure 5, indicating that all the poles are stable with sufficient damping.

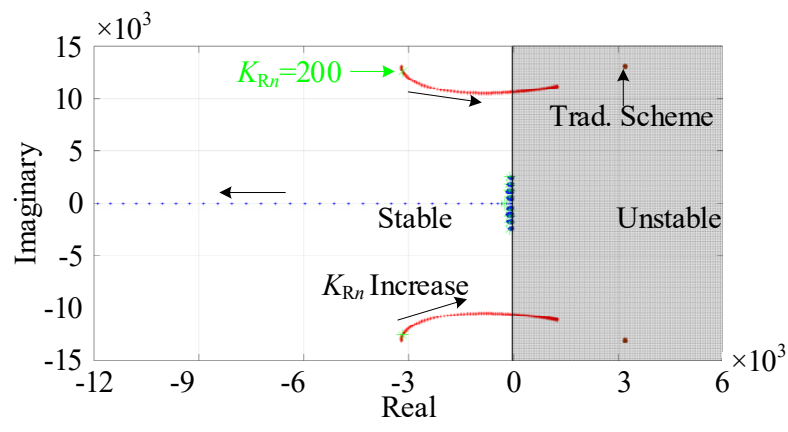

Figure 5. Pole locus of $H_{\text {is }}(s)$ as a function of $K_{\mathrm{R} n}$, where the proposed modulation scheme and control strategy are applied. The poles with the traditional schemes are also shown for comparison.

The influence can also be found from the expression of $Y_{\text {id }}(s)$ shown in Equation (29). When $K_{R n}$ is small, the gain of $H_{\mathrm{y}}(s)$ at the resonant frequency is small and thus the new modulation scheme dominates, enhancing the stability. On the contrary, when $K_{\mathrm{R} n}$ is extremely large, $H_{\mathrm{y}}(s)$ can be considered at unity. In this case, $Y_{\text {id }}$ is negative, just as for the one generated by the traditional modulation schemes. This means that the new modulation scheme and the proposed feedback control strategy will behave like the traditional modulation schemes under extremely large values of $K_{\mathrm{R} n}$. Nevertheless, small $K_{\mathrm{R} n}$ values are always sufficient to eliminate the effects of input voltage disturbances, and thus the influence of the additional feedback control strategy on the UMC stability is quite minor.

\section{Experimental Verification}

Experimental verification is conducted on the UMC prototype shown in Figure 6. The prototype parameters are listed in Table 1. The typical closed-loop control of output phase currents in a stationary reference frame is applied to generate the reference amplitude and phase angle of output voltages. A high-performance programmable AC source (Chroma 61511) serves as the input source.

Firstly, source voltages are set as balanced and sinusoidal. The root mean square (RMS) value of phase voltages is $100 \mathrm{~V}$. The experimental result with the traditional modulation scheme shown in Figure $7 \mathrm{a}$ indicates that the UMC becomes unstable with severe filter resonance observed. Both the input and output power quality are very poor. It should be noted that the output current amplitude reference is reduced by half in this case. If the reference is set to the rated value listed in Table 1 , the stability will be even worse. As a result, an additional stabilization method is indispensable for the traditional scheme. When the proposed modulation scheme is applied instead, both the input and output currents are highly sinusoidal, as shown in Figure $7 \mathrm{~b}$. In particular, the input filter resonance is suppressed effectively without an additional passive or active stabilization method. The proposed scheme is quite suitable for applications with sinusoidal and balanced input voltages, such as the distributed generation system with a generator input. In these applications, the proposed scheme is preferable since the elimination of an additional stabilization method could reduce the control and hardware complexity of the UMC significantly. Therefore, there will be more space, in both the software and hardware, to achieve other control objectives. 


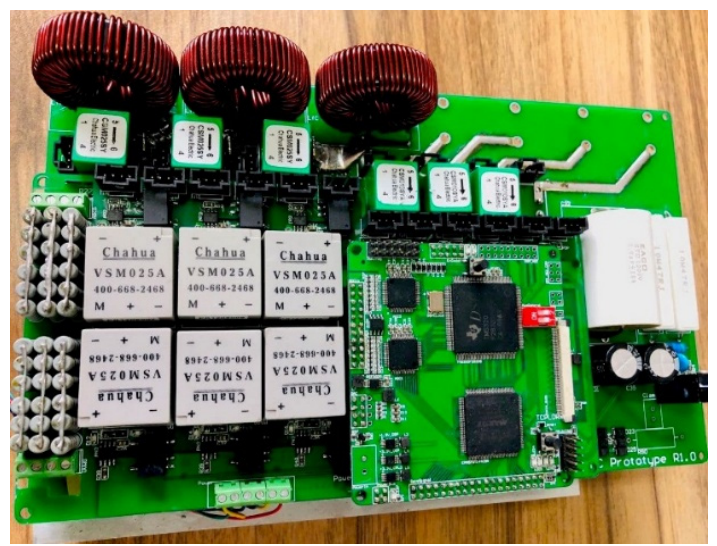

Figure 6. Experimental prototype of the unidirectional matrix converter.

Table 1. Parameters of the UMC prototype.

\begin{tabular}{cc}
\hline Term & Value \\
\hline Input Filter Inductor, $L_{\mathrm{f}}$ & $1.1 \mathrm{mH}$ \\
Input Filter Capacitor, $C_{\mathrm{f}}$ & $5.0 \mu \mathrm{F}$ \\
Resistance of $L_{\mathrm{f}}$ & $0.01 \Omega$ \\
Output Inductor, $L_{\mathrm{o}}$ & $10.6 \mathrm{mH}$ \\
Output Resistor, $R_{\mathrm{o}}$ & $10.0 \Omega$ \\
Output Current Amplitude Reference, & $8.0 \mathrm{~A}$ \\
$i_{\text {om }}{ }^{*}$ & $50 \mathrm{~Hz}$ \\
Input Frequency & $60 \mathrm{~Hz}$ \\
Output Frequency, $f_{\mathrm{o}}$ & $30 \mathrm{kHz}$ \\
Sampling Frequency, $f_{\mathrm{s}}$ & 200 \\
Static Gain, $K_{\mathrm{R} n}$, of Each RC &
\end{tabular}

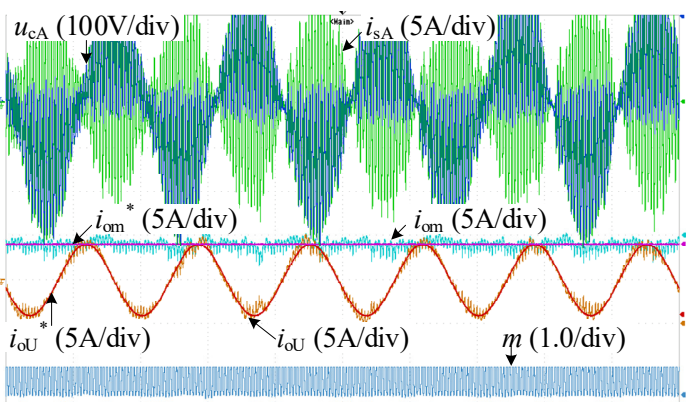

Time (10ms/div)

(a)

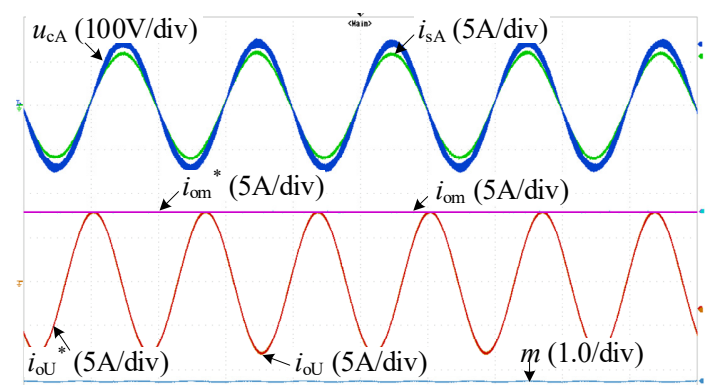

Time $(10 \mathrm{~ms} / \mathrm{div})$

(b)

Figure 7. Experimental results under balanced and sinusoidal input voltages: (a) with traditional modulation scheme; (b) with the proposed modulation scheme.

Secondly, source voltages are set as unbalanced and distorted. The RMS values of the three-phase source voltages are $120 \mathrm{~V}, 100 \mathrm{~V}$, and $80 \mathrm{~V}$, respectively, causing voltage unbalance. Each phase voltage contains $5 \% 5^{\text {th }}$ and $5 \% 7^{\text {th }}$ harmonics. Therefore, the source voltages are highly disturbed, as shown in Figure 8. Figure 9a shows the experimental results with the proposed modulation scheme and without the proposed feedback control strategy. It is clear that only the proposed modulation scheme cannot obtain sinusoidal output currents in this case. Significant low-frequency ripples are observed in the instantaneous amplitude, $i_{\text {om }}$, of output currents. The output current is highly distorted. It should be noted that the traditional modulation schemes with an additional stabilization method based on input voltage filtering also suffer from degraded power quality under input disturbances [20]. The proposed feedback control strategy is then incorporated, of which the experimental results are 
shown in Figure 9b. It can be seen that the output current is highly sinusoidal and perfectly tracks its reference. The low-frequency ripples in its amplitude, $i_{\mathrm{om}}$, are eliminated. The additional control signal $y$ generated by the proposed feedback control strategy contributes to the variation of the modulation index $m$, which compensates the source voltage disturbances and thus is the reason for the high output power quality. In addition, the filter resonance is still effectively suppressed, as shown in Figure $9 \mathrm{a}, \mathrm{b}$, which proves that the proposed modulation scheme can always enhance the MC stability, regardless of the source voltage disturbances. To sum up, although the proposed modulation scheme reduces the immunity of the UMC to the input voltage disturbances, the proposed feedback control strategy can easily suppress such side effect. Therefore, the proposed modulation scheme and control strategy is a preferable solution for general applications, even with large source voltage disturbances, considering the prominent advantage of increased stability.

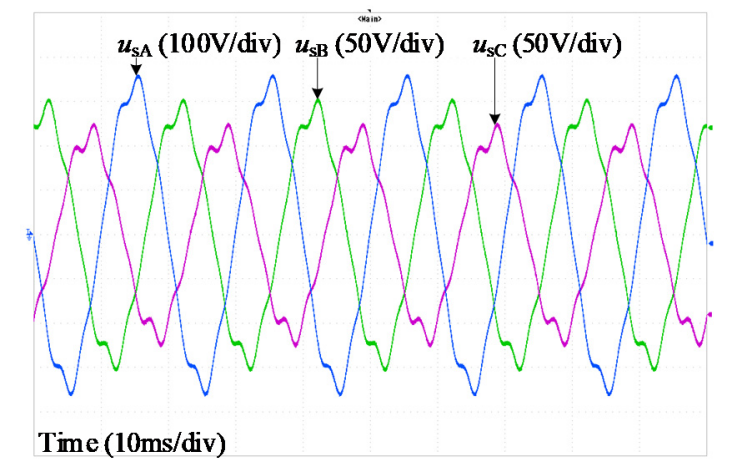

Figure 8. Waveforms of the three-phase unbalanced and distorted source voltages.

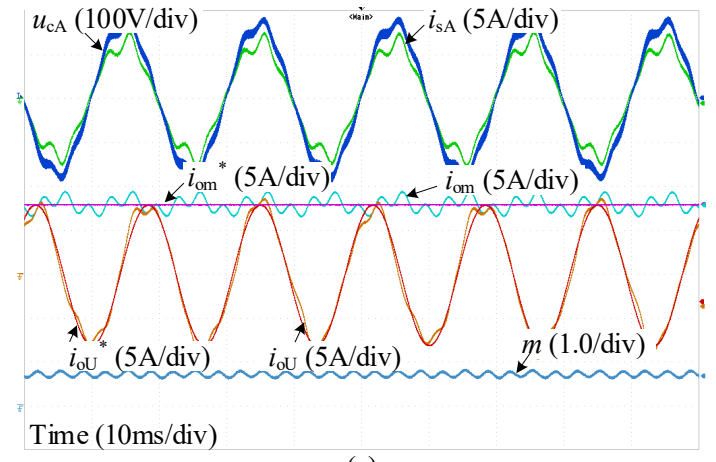

(a)

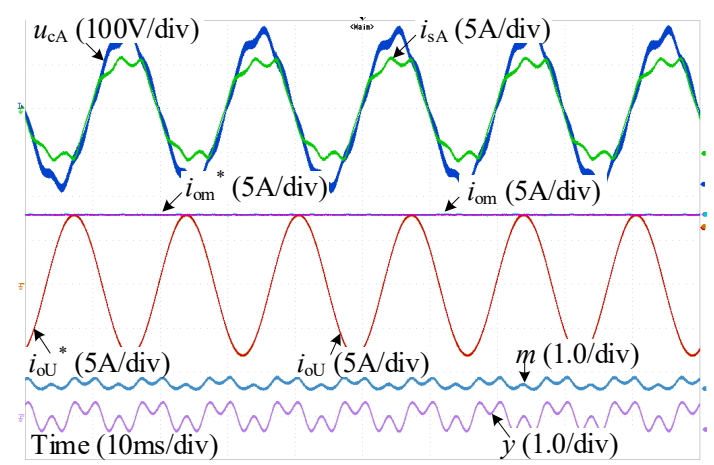

(b)

Figure 9. Experimental results with the proposed modulation scheme under disturbed input condition: (a) without the proposed feedback control strategy; (b) with the proposed feedback control strategy.

The dynamic performance of the proposed modulation scheme and feedback control strategy is also evaluated. The obtained experimental results are shown in Figure 10. In Figure 10a, the reference amplitude of output current fluctuates between $8 \mathrm{~A}$ and $4 \mathrm{~A}$. It is clear that the additional control signal $y$ is quite small during the dynamic process, which indicates that the proposed control solution does not influence the dynamic performance when the load changes. This is also demonstrated by the experimental results shown in Figure 10a. In Figure 10b, the amplitude of source voltages is increased by $10 \%$ and then returns to the normal. Although the amplitude of output current is affected slightly during this dynamic process, the proposed feedback control strategy together with the typical closed-loop control of output currents can effectively suppress the effects of the disturbances in a short time. Moreover, the sudden change of the input voltage amplitude has minor influence on the additional control signal $y$, which remains small during the dynamic process. Figure 10 proves that the proposed control solution could achieve satisfactory dynamic performance considering the source and load transients. 


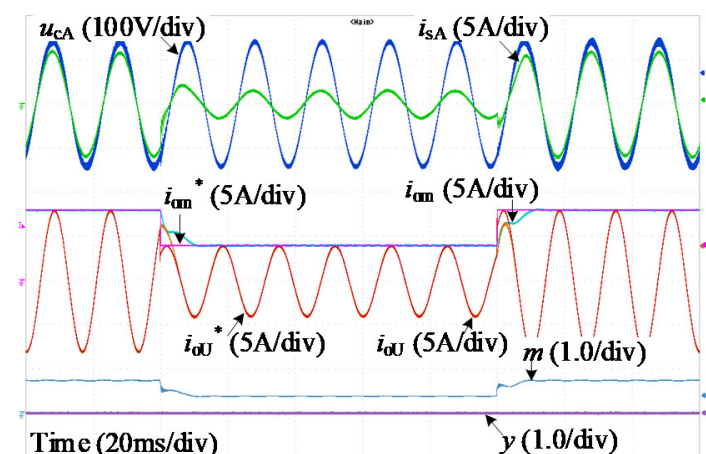

(a)

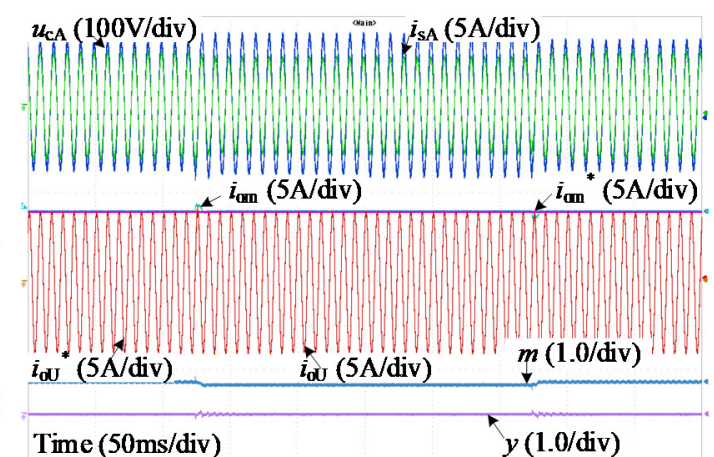

(b)

Figure 10. Experimental results with the proposed modulation scheme and feedback control strategy during the dynamic process: (a) the reference amplitude, $i_{\mathrm{om}}{ }^{*}$, of output current fluctuates between $8 \mathrm{~A}$ and $4 \mathrm{~A} ;(\mathbf{b})$ the amplitude of source voltages is increased by $10 \%$ and then returns to the normal.

\section{Conclusions}

Input disturbance suppression and stability improvement are two important issues for the UMC, both of which are highly dependent on the modulation scheme. Although the existing modulation schemes could theoretically suppress the input disturbances, they suffer from the degraded stability. On the contrary, the modulation scheme proposed in this paper always enhances the stability of the UMC, especially under high active power output, which is well demonstrated by experimental results. For applications with sinusoidal and balanced source voltages, the proposed modulation scheme is preferable since the hardware and control complexity is reduced significantly. When source voltages are disturbed, the proposed feedback control strategy could effectively eliminate the output low-frequency harmonics without affecting the stability performance. Therefore, the proposed control solution is a promising alternative to the existing control solutions for the UMC.

The new modulation scheme is not suitable for the standard MC working in reverse mode. In this case, the traditional modulation schemes with filtered input phase angle can be applied instead, which will not generate additional low-frequency harmonics in the output currents. Therefore, a hybrid control solution is suitable for the standard MC.

Author Contributions: Conceptualization, J.L. and S.F.; methodology, J.L.; software, C.W.; validation, C.W.; formal analysis, J.L.; investigation, C.W.; resources, J.L. and S.F.; data curation, C.W.; writing一 original draft preparation, J.L.; writing-review and editing, S.F.; visualization, C.W.; supervision, J.L. and S.F.; project administration, J.L. and S.F.; funding acquisition, J.L. and S.F.

Funding: This work was supported in part by the National Natural Science Foundation of China under Grant 51807025 and in part by the Natural Science Foundation of Jiangsu Province of China under Grant BK20180396.

Conflicts of Interest: The authors declare no conflict of interest.

\section{Appendix A}

(1) Derivation of the dq-axis input admittances in Equations (13) and (14)

With the substitution of Equation (12) into Equations (10) and (11), the dq-axis input currents can be expressed as

$$
\begin{aligned}
& i_{\mathrm{id}}=\frac{p_{\mathrm{o}}\left(u_{\mathrm{cd}}+u_{\mathrm{cq}} \tan \varphi_{\mathrm{i}}\right)}{1.5\left(u_{\mathrm{cd}}^{2}+u_{\mathrm{cq}}^{2}\right)}, \\
& i_{\mathrm{id}}=\frac{p_{\mathrm{o}}\left(u_{\mathrm{cd}}+u_{\mathrm{cq}} \tan \varphi_{\mathrm{i}}\right)}{1.5\left(u_{\mathrm{cd}}^{2}+u_{\mathrm{cq}}^{2}\right)} .
\end{aligned}
$$


For the balanced and sinusoidal load, the active power, $p_{0}$, is a constant signal and is independent of the input voltages. Therefore, according to the definition of the input admittances, $Y_{\text {id }}$ and $Y_{\text {iq }}$ can be expressed as

$$
\begin{aligned}
& Y_{\mathrm{id}}=\frac{\partial i_{\mathrm{id}}}{\partial u_{\mathrm{cd}}}=\frac{p_{\mathrm{o}}}{1.5} \frac{\left(U_{\mathrm{cd}}^{2}+U_{\mathrm{cq}}^{2}\right)-2 U_{\mathrm{cd}}\left(U_{\mathrm{cd}}+U_{\mathrm{cq}} \tan \varphi_{\mathrm{i}}\right)}{\left(U_{\mathrm{cd}}^{2}+U_{\mathrm{cq}}^{2}\right)^{2}}, \\
& Y_{\mathrm{iq}}=\frac{\partial i_{\mathrm{iq}}}{\partial u_{\mathrm{cq}}}=\frac{p_{\mathrm{o}}}{1.5} \frac{\left(U_{\mathrm{cd}}^{2}+U_{\mathrm{cq}}^{2}\right)-2 U_{\mathrm{cq}}\left(U_{\mathrm{cq}}-U_{\mathrm{cd}} \tan \varphi_{\mathrm{i}}\right)}{1.5\left(U_{\mathrm{cd}}^{2}+U_{\mathrm{cq}}^{2}\right)^{2}} .
\end{aligned}
$$

For the synchronous reference frame oriented with the source voltage vector, the capacitor voltages satisfy

$$
U_{\mathrm{cd}} \approx U_{\mathrm{cm}}, U_{\mathrm{cq}} \approx 0 \text {. }
$$

Besides, the input power factor of the MC is usually set to unity, which means

$$
\tan \varphi_{\mathrm{i}} \approx 0 .
$$

By substituting Equations (A5) and (A6) into Equations (A3) and (A4), expressions of the dq-axis input admittances shown in Equations (13) and (14) can be obtained.

(2) Derivation of the dq-axis input admittances in Equations (29) and (30)

Similarly, the dq-axis input admittances with the proposed control strategy are derived from the expressions of input currents shown in Equations (27) and (28):

$$
\begin{gathered}
Y_{\mathrm{id}}=\frac{\partial i_{\mathrm{id}}}{\partial u_{\mathrm{cd}}}=\frac{p_{\mathrm{o}}}{1.5 U_{\mathrm{cm}}^{2}}\left[\frac{1}{1-Y}+\frac{U_{\mathrm{cd}}+U_{\mathrm{cq}} \tan \varphi_{\mathrm{i}}}{(1-Y)^{2}} \frac{\partial y}{\partial u_{\mathrm{cd}}}\right], \\
Y_{\mathrm{iq}}=\frac{\partial i_{\mathrm{iq}}}{\partial u_{\mathrm{cq}}}=\frac{p_{\mathrm{o}}}{1.5 U_{\mathrm{cm}}^{2}}\left[\frac{1}{1-Y}+\frac{U_{\mathrm{cq}}-U_{\mathrm{cd}} \tan \varphi_{\mathrm{i}}}{(1-Y)^{2}} \frac{\partial y}{\partial u_{\mathrm{cq}}}\right],
\end{gathered}
$$

where $Y$ is the value of $y$ at the steady state, which should be zero; $p_{o}$ is still assumed to be a constant signal under balanced and sinusoidal outputs. Therefore, with the substitution of Equations (A3) and (A4), Equations (A5) and (A6) are simplified as

$$
\begin{gathered}
Y_{\mathrm{id}}=\frac{\partial i_{\mathrm{id}}}{\partial u_{\mathrm{cd}}}=\frac{p_{\mathrm{o}}}{1.5 U_{\mathrm{cm}}^{2}}\left(1+U_{\mathrm{cd}} \frac{\partial y}{\partial u_{\mathrm{cd}}}\right), \\
Y_{\mathrm{iq}}=\frac{\partial i_{\mathrm{iq}}}{\partial u_{\mathrm{cq}}}=\frac{p_{\mathrm{o}}}{1.5 U_{\mathrm{cm}}^{2}} .
\end{gathered}
$$

It can be seen that the proposed control strategy only affects the d-axis input admittances. According to Figure $3 b$, variable $y$ satisfies

$$
\ell(y)=H_{\mathrm{y}}(s) \cdot \ell\left(1-\frac{u_{\mathrm{cm}}^{2}}{U_{\mathrm{cm}}^{2}}\right),
$$

where $\ell$ represents the Laplace transformation. Equation (A12) is then derived from Equation (A11):

$$
\frac{\partial y}{\partial u_{\mathrm{cd}}}=-\frac{2 H_{\mathrm{y}}(s)}{U_{\mathrm{cm}}}
$$

By substituting Equations (A5) and (A12) into Equations (A9) and (A10), expressions of the input admittances in Equations (29) and (30) can thus be obtained. 


\section{References}

1. Empringham, L.; Kolar, J.W.; Rodriguez, J.; Wheeler, P.W.; Clare, J.C. Technological Issues and Industrial Application of Matrix Converters: A Review. IEEE Trans. Ind. Electron. 2013, 60, 4260-4271. [CrossRef]

2. Rząsa, J. An Alternative Carrier-Based Implementation of Space Vector Modulation to Eliminate Common Mode Voltage in a Multilevel Matrix Converter. Electronics 2019, 8, 190. [CrossRef]

3. Song, W.; Liu, J.; Sun, X.; Wu, F.; Gao, D.; Wang, Y. Research on Commutation and Coordination Control Strategy of Excitation Power Supply Based on Bidirectional Reduced Matrix Converter for Ion Accelerator. Energies 2018, 11, 3396. [CrossRef]

4. Merchan-Villalba, L.; Lozano-Garcia, J.; Gutierrez-Torres, D.; Avina-Cervantes, J.; Pizano-Martinez, A. Four-Step Current Commutation Strategy for a Matrix Converter Based on Enhanced-PWM MCU Peripherals. Electronics 2019, 8, 547. [CrossRef]

5. Szczesniak, P. Challenges and Design Requirements for Industrial Applications of AC/AC Power Converters without DC-Link. Energies 2019, 12, 1581. [CrossRef]

6. Kolar, J.W.; Schafmeister, F.; Round, S.D.; Ertl, H. Novel Three-Phase AC-AC Sparse Matrix Converters. IEEE Trans. Power Electron. 2007, 22, 1649-1661. [CrossRef]

7. Xia, C.; Li, S.; Yan, Y.; Shi, T. Research on Linear Output Voltage Transfer Ratio for Ultrasparse Matrix Converter. IEEE Trans. Power Electron. 2016, 31, 1811-1815. [CrossRef]

8. Shi, T.; Zhang, X.; An, S.; Yan, Y.; Xia, C. Harmonic Suppression Modulation Strategy for Ultra-Sparse Matrix Converter. IET Power Electron. 2016, 9, 589-599. [CrossRef]

9. Bozorgi, A.M.; Hakemi, A.; Farasat, M.; Monfared, M. Modulation Techniques for Common-Mode Voltage Reduction in the Z-Source Ultra Sparse Matrix Converters. IEEE Trans. Power Electron. 2019, 34, 958-970. [CrossRef]

10. Rodriguez, J.; Rivera, M.; Kolar, J.W.; Wheeler, P.W. A Review of Control and Modulation Methods for Matrix Converters. IEEE Trans. Ind. Electron. 2012, 59, 58-70. [CrossRef]

11. Li, S.; Xia, C.; Yan, Y.; Shi, T. Space-Vector Overmodulation Strategy for Ultrasparse Matrix Converter Based on the Maximum Output Voltage Vector. IEEE Trans. Power Electron. 2017, 32, 5388-5397. [CrossRef]

12. Nguyen, H.; Lee, H. An Effective SVM Method for Matrix Converters with a Superior Output Performance. IEEE Trans. Ind. Electron. 2018, 65, 6948-6958. [CrossRef]

13. Tsoupos, A.; Khadkikar, V. A Novel SVM Technique with Enhanced Output Voltage Quality for Indirect Matrix Converters. IEEE Trans. Ind. Electron. 2019, 66, 832-841. [CrossRef]

14. Lei, J.; Zhou, B.; Qin, X.; Wei, J.; Bian, J. Active Damping Control Strategy of Matrix Converter Via Modifying Input Reference Currents. IEEE Trans. Power Electron. 2015, 30, 5260-5271. [CrossRef]

15. Casadei, D.; Serra, G.; Tani, A.; Trentin, A.; Zarri, L. Theoretical and Experimental Investigation on the Stability of Matrix Converters. IEEE Trans. Ind. Electron. 2005, 52, 1409-1419. [CrossRef]

16. Sato, I.; Itoh, J.; Ohguchi, H.; Odaka, A.; Mine, H. An Improvement Method of Matrix Converter Drives Under Input Voltage Disturbances. IEEE Trans. Power Electron. 2007, 22, 132-138. [CrossRef]

17. Sun, Y.; Su, M.; Li, X.; Wang, H.; Cui, W. A General Constructive Approach to Matrix Converter Stabilization. IEEsE Trans. Power Electron. 2013, 28, 418-431. [CrossRef]

18. Cardenas, R.; Pena, R.; Tobar, G.; Clare, J.; Wheeler, P.; Asher, G. Stability Analysis of a Wind Energy Conversion System Based on a Doubly Fed Induction Generator Fed by a Matrix Converter. IEEE Trans. Ind. Electron. 2009, 56, 4194-4206. [CrossRef]

19. Lei, J.; Zhou, B.; Qin, X.; Bian, J.; Wei, J. Stability Improvement of Matrix Converter by Digitally Filtering the Input Voltages in Stationary Frame. IET Power Electron. 2016, 9, 743-750. [CrossRef]

20. Lei, J.; Feng, S.; Zhou, B.; Cao, W.; Zhao, J. Evaluation and Improvement of Active Stabilization Method for Matrix Converter under Input Voltage Disturbances. IEEE J. Emerg. Sel. Top. Power Electron. 2019, 7, 1116-1125. [CrossRef]

(C) 2019 by the authors. Licensee MDPI, Basel, Switzerland. This article is an open access article distributed under the terms and conditions of the Creative Commons Attribution (CC BY) license (http://creativecommons.org/licenses/by/4.0/). 